\title{
Ensino da Língua Brasileira de Sinais nos cursos de graduação em Odontologia do Sudeste brasileiro: um estudo transversal
}

\author{
Yuri de Lima Medeiros*; Danielle Fernandes Lopes*; Luan Viana Faria*, Mônica Regina Pereira Senra \\ Soares**; Carla Couto de Paula Silvério***
}
* Discente, Curso de Odontologia, Universidade Federal de Juiz de Fora
** Doutora, Professora Adjunta, Departamento de Odontologia, da Universidade Federal de Juiz de Fora - Campus Avançado de Governador Valadares
*** Doutoranda, Programa de Pós-Graduação em Linguística, Vice Coordenadora, Curso de Letras/Libras, Universidade Federal de Juiz de Fora

Recebido em 23/08/2019. Aprovado em 30/04/2020.

\begin{abstract}
RESUMO
$\mathrm{O}$ atendimento odontológico de pacientes surdos é considerado um desafio. A falta de contato dos estudantes com a Língua Brasileira de Sinais (Libras) durante a graduação pode ser um fator agravante da condição de saúde bucal desta população. Assim, o objetivo do estudo foi oferecer um panorama sobre a inclusão da disciplina de Libras nos projetos pedagógicos dos cursos de graduação em Odontologia da Região Sudeste do Brasil. Consiste em um estudo exploratório, quantitativo e transversal. As matrizes curriculares dos cursos foram analisadas, buscando-se as informações referentes às variáveis: categoria administrativa da Instituição de Ensino Superior (IES), oferta e natureza do componente curricular e carga horária. A população do estudo foi constituída por 176 IES, das quais 32 não disponibilizaram suas respectivas matrizes curriculares. Observou-se que 125 cursos $(86,8 \%)$ eram de IES particulares e $19(13,19 \%)$ de públicas, com 83 (57,63\%) oferecendo o componente curricular Libras em sua matriz, sendo que $71(91,02 \%)$ das 78 IES que disponibilizaram a informação ofertam o conteúdo como opcional ou eletivo. A carga horária variou de 30 a 80 horas, com média de 49,7h. Conclui-se que a implementação da Libras ainda é discreta nas universidades públicas e mais concentrada nas IES privadas, sendo, em sua maioria, não-obrigatória.
\end{abstract}

Descritores: Educação em Odontologia. Línguas de Sinais. Currículo. 


\section{INTRODUÇÃO}

De acordo com o último censo realizado pelo Instituto Brasileiro de Geografia e Estatística (IBGE), em 2010, 9,8 milhões de brasileiros possuem algum tipo de surdez, representando $5,2 \%$ da população brasileira. Deste total, 2,6 milhões são surdos e 7,2 milhões apresentam grande dificuldade para ouvir ${ }^{1}$. Vale dizer que as pessoas com surdez podem ser divididas em dois grandes grupos: (i) o das pessoas com deficiência auditiva, que possuem alguma perda auditiva em diferentes níveis, mas não se sente confortável em usar a língua de sinais de seu país, usando a língua oral; e (ii) o das pessoas surdas que utilizam a língua de sinais de seu país como principal meio de comunicação².

Para o atendimento dos pacientes surdos, em 2005, o Decreto $n^{\circ} 5.626 / 05^{3}$ regulamentou a Lei n. ${ }^{\circ} 10.436 / 02^{4}$ e propôs uma adequação curricular a partir das novas políticas inclusivas nas universidades. Esse decreto reconhece a Língua Brasileira de Sinais (Libras) como língua oficial da comunidade surda e a coloca como disciplina obrigatória nas licenciaturas gerais e nos cursos de fonoaudiologia. Essa legislação foi, sem dúvida, uma grande conquista política para a comunidade surda.

Paralelamente, as Diretrizes Curriculares Nacionais (DCN) para o curso de Odontologia, reformuladas e aprovadas no Conselho Nacional de Educação em 2018 ${ }^{5}$, adotaram como referência, dentre outras, a Educação em Saúde e as Novas Tecnologias de Informação e Comunicação em Odontologia, inclusive as línguas oficiais do território brasileiro (Língua Portuguesa e Libras) como componentes curriculares. No entanto, o documento não especifica os aspectos referentes à carga horária e ementa da disciplina de Libras. Contudo, enfatiza que o egresso de Odontologia deve estar atento à dignidade da pessoa humana e às suas necessidades individuais. Dentre as competências ele deverá ser capaz de mobilizar recursos para solucionar, com sucesso, os problemas da prática profissional em diferentes contextos; sendo o sujeito da sua própria aprendizagem com vistas à sua formação integral e adequada articulando o ensino, pesquisa e a extensão em prol da preservação de culturas e práticas nacionais e regionais respeitando o pluralismo de concepções e a diversidade étnicacultural.

Sabe-se que a disciplina de Libras na formação do estudante de Odontologia pode beneficiar a comunicação com os pacientes surdos falantes da língua de sinais de forma diferenciada ${ }^{6}$. Existe a demanda para o atendimento odontológico de pacientes surdos, porém a barreira de comunicação se traduz em um grande desafio. Associa-se a esse fato a escassez de profissionais habilitados, a dificuldade de acesso aos serviços de saúde e a concepção negativa do atendimento odontológico $^{7}$. Consequentemente, a condição de saúde bucal desses pacientes é agravada, apresentando, por exemplo, maior prevalência de cárie dentária e higiene oral deficiente, quando comparados aos pacientes ouvintes ${ }^{7-9}$.

Frente ao exposto, este estudo tem como objetivo oferecer um panorama sobre a inclusão da disciplina de Libras nos projetos pedagógicos dos cursos de graduação em Odontologia da Região Sudeste, que apresenta a maior concentração de cursos de Odontologia do Brasil $^{10}$.

\section{METODOLOGIA}

A presente pesquisa consiste em um estudo exploratório, quantitativo e transversal. Os cursos foram identificados na base de dados oficial de informações relativas às Instituições de Ensino Superior (IES), o Cadastro Nacional de Cursos e Instituições de Educação Superior

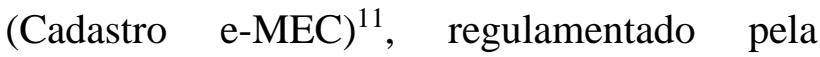


Portaria Normativa $\mathrm{n}^{\mathrm{o}} 21$, de 21 de dezembro de $2017^{12}$, que pode ser acessado no endereço eletrônico do Instituto Nacional de Estudos e Pesquisas Educacionais Anísio Teixeira (INEP).

A população do estudo consistiu em todos os cursos de Odontologia em funcionamento na Região Sudeste ( $\mathrm{n}=176)$ no ano de 2019, assim distribuídos: Espírito Santo (ES, $\mathrm{n}=12)$, Minas Gerais (MG, n=61), Rio de Janeiro (RJ, n=28) e São Paulo (SP, n=75). Foram excluídos aqueles que não possuíam um sítio web oficial e não forneciam meios de comunicação virtual. Foram incluídos na pesquisa todos aqueles que apresentaram sua matriz curricular completa disponível.

Para confirmar a existência da disciplina de Libras, realizou-se a pesquisa em todos os sítios web institucionais, complementada, quando necessário, por contato com os coordenadores. Foi disponibilizado um prazo de dois meses para que os e-mails fossem respondidos. O período de coleta dos dados foi de fevereiro a junho de 2019.

Foram coletadas as seguintes informações sobre a disciplina de Libras: (i) categoria administrativa da IES (pública ou privada), (ii) inserção e oferta da disciplina, (iii) natureza do componente curricular (obrigatória ou nãoobrigatória) e (iv) carga horária total.

Os dados foram organizados e tabulados utilizando o programa GraphPad Prism 8.1.2 Realese notes (La Jolla, CA, EUA) sendo analisados por meio de estatísticas descritivas.

\section{RESULTADOS}

Dos 176 cursos, considerando os polos de uma mesma instituição, 32 não disponibilizaram a matriz curricular, de forma que a amostra final foi composta por 144 cursos $(81,8 \%), 10(6,94 \%)$ do estado do ES, 51 (35,41\%) em MG, 21 $(14,58 \%)$ no RJ e $62(43,05 \%)$ em SP.

Observou-se que, quanto à categoria administrativa, 125 cursos $(86,8 \%)$ eram de IES particulares e 19 cursos $(13,19 \%)$ eram de IES públicas. Dentre todas as IES que ofertam as disciplinas de Libras, 76 (91,56\%) são privadas e $7(8,43 \%)$ públicas. Do total das 144 IES, 83 $(57,63 \%)$ ofertam a disciplina de Libras em sua matriz curricular (tabela 1 ).

Tabela 1. Oferta da disciplina de Libras nos cursos de Odontologia das IES públicas e privadas dos estados do Sudeste brasileiro $(n=144)$

\begin{tabular}{l|cccc}
\hline \multirow{2}{*}{ Libras como componente curricular } & \multicolumn{4}{|c}{ Estados } \\
\hline Instituições Públicas $(\mathrm{n}=19 / 13,19 \%)$ & ES & MG & RJ & SP \\
Sim $(\mathrm{n}=7 / 36,8 \%)$ & 1 & 4 & 2 & 0 \\
Não $(\mathrm{n}=12 / 63,1 \%)$ & 0 & 3 & 2 & 7 \\
Instituições Privadas $(n=125 / 86,8 \%)$ & & & & \\
Sim ( $\mathrm{n}=76 / 60,8 \%)$ & 7 & 26 & 10 & 33 \\
Não $(\mathrm{n}=49 / 39,2 \%)$ & 2 & 18 & 7 & 22 \\
\hline
\end{tabular}

Em relação à natureza do componente curricular, 5 (6,02\%) IES não disponibilizaram a informação. Dentre as 78 IES que a disponibilizaram, $71(91,02 \%)$ ofertam o conteúdo de forma opcional ou eletiva, enquanto apenas $7(8,97 \%)$ apresentam de forma obrigatória (gráfico 1). A carga horária foi disponibilizada por $61(73,49 \%)$ IES, variando de 30 a 80 horas, com média de 49,54h. Dentre os estados, o ES apresentou a maior média, enquanto que MG apresentou a menor (gráfico 2). 


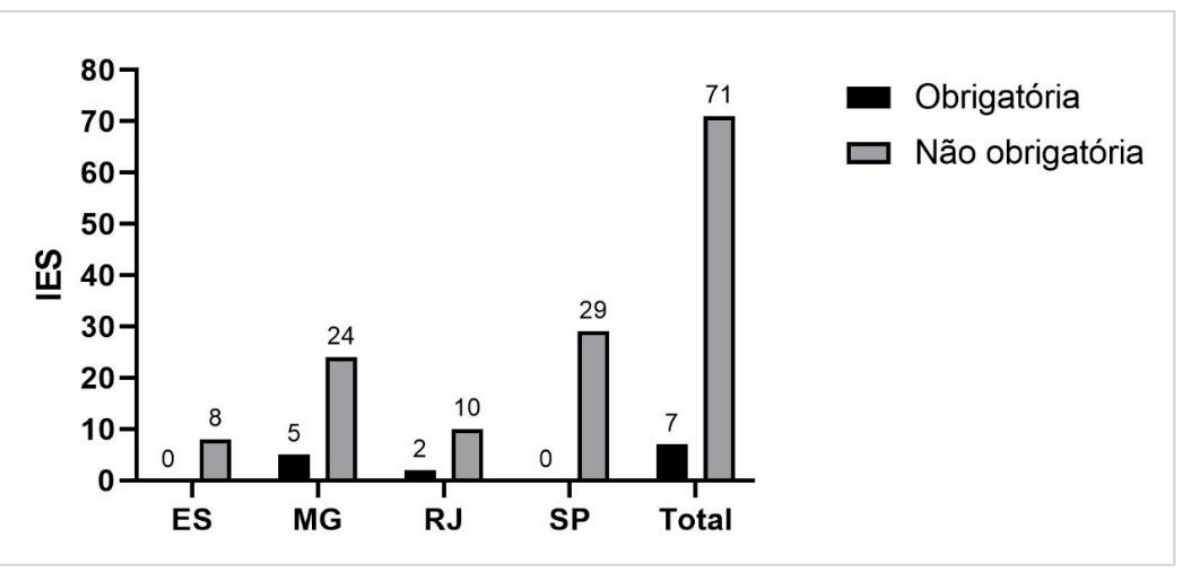

Gráfico 1. Natureza do componente curricular de Libras nos cursos de Odontologia dos estados do Sudeste brasileiro

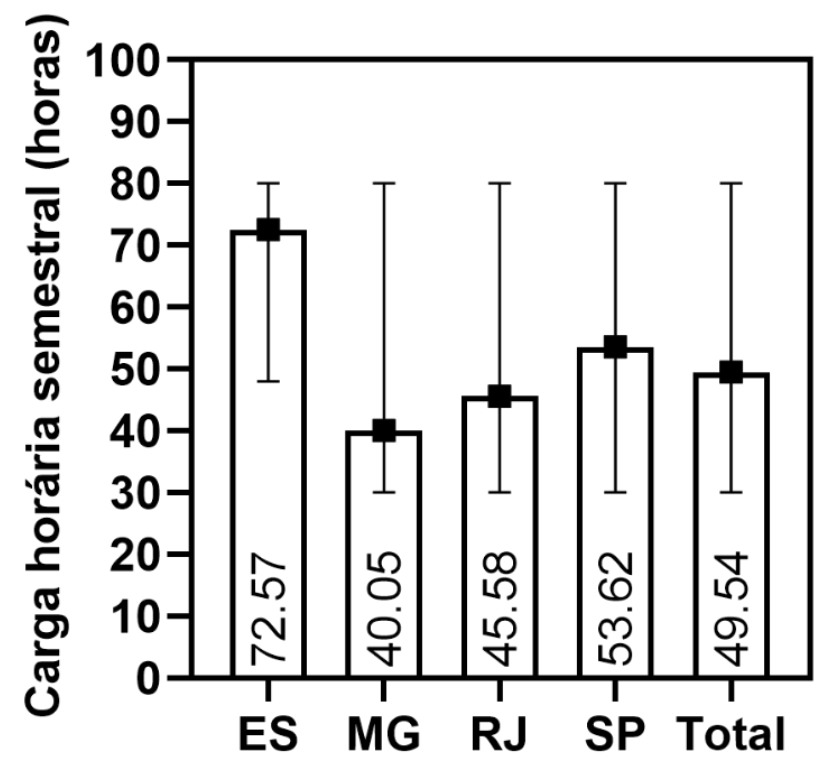

Gráfico 2. Carga horária média da disciplina de Libras na matriz curricular dos cursos de Odontologia dos estados do Sudeste brasileiro

\section{DISCUSSÃO}

De acordo com os resultados obtidos, observou-se um maior número de IES privadas em relação às públicas na região Sudeste do Brasil. Das instituições públicas ( $\mathrm{n}=19)$ apenas $36,84 \%$ apresentam a disciplina de Libras no conteúdo curricular, sendo que em SP, maior estado em termos populacionais do Brasil $^{13}$, nenhuma a oferece. Há inversão destes dados nos cursos particulares $(60,8 \%)$, o que está de acordo com os achados de Oliveira et al. ${ }^{14}$, que trazem informações de que as instituições privadas têm buscado atender à determinação legal e às recomendações do Ministério da Educação. As $\mathrm{DCN}^{6}$ para o curso de Odontologia trazem esse conteúdo como componente curricular obrigatório, 
independente do caráter que ela poderá assumir, havendo prazo de até dois anos, a partir da data de publicação da Resolução, para aplicação dessa determinação às novas turmas abertas. Para Martins $^{6}$, há que se ter o cuidado para não tornar superficial o ensino de Libras, por apresentar-se como única disciplina semestral. Enfatiza que uma educação bilingue ultrapassa os limites das salas de aula, necessitando de maior contato com a cultura em questão.

A oferta dessa disciplina nos cursos de Odontologia já se traduz em um grande passo para a humanização e integralidade da assistência, tendo em vista garantir ao estudante conhecer o processo de comunicação de indivíduos com surdez, além de promover o ensejo dos graduandos em aperfeiçoar, por meio de técnicas adequadas, essa comunicação interpessoal com vistas a atender as suas demandas em saúde.

Em relação à natureza do componente curricular, mais de $90 \%$ das IES ofertam enquanto disciplina eletiva/opcional e apenas uma pequena parte oferta enquanto disciplina obrigatória. Segundo o decreto $5.626 / 05^{3}$, somente os cursos de magistério e Fonoaudiologia devem ofertar a disciplina de Libras como um componente obrigatório do currículo, sendo que os demais cursos, como o de Odontologia, podem ofertar como eletiva ou opcional. Isso mostra coerência na organização dos currículos. Entretanto, entende-se que a necessidade do conhecimento da Libras e da cultura surda é importante para oferecer um atendimento ao público surdo de maneira acessível e humanizada ${ }^{14,15}$.

A principal razão que afasta ou coloca os surdos em experiências negativas nos atendimentos em saúde é a barreira comunicativa, justificada pela falta de capacitação e conscientização dos profissionais ${ }^{15-17}$. Em relação ao ambiente odontológico esse problema fica ainda mais visível, gerando comportamentos de medo e ansiedade previamente aos procedimentos ${ }^{18}$. Assim, vencer essa barreira, com a oferta da disciplina de Libras de forma obrigatória na matriz curricular da graduação, favorece uma aproximação com o tema, sensibilizando o estudante a rever as práticas de cuidado na assistência integral e humanizada ao paciente com surdez.

Pereira et al. ${ }^{19}$ realizaram uma pesquisa com aplicação de questionário estruturado para pacientes surdos e 70\% consideraram que os cirurgiões-dentistas não estão preparados para atendê-los, sendo que $56,6 \%$ da amostra se manifestou insatisfeita com suas experiências odontológicas anteriores. Os dados encontrados corroboram outras pesquisas ${ }^{20-22}$, que demonstraram a insatisfação dos pacientes surdos quanto aos serviços prestados, principalmente no que se relaciona à dificuldade de comunicação.

No estudo de Alshehri et al. ${ }^{20}$ verificou-se que o método de comunicação mais utilizado pelos surdos é a língua de sinais, seguido pela sua combinação com a leitura labial. Contudo, a maioria da amostra $(72,1 \%)$ relatou que nunca foi questionada pela equipe odontológica sobre qual forma de comunicação utilizavam. Embora a língua de sinais seja a forma mais utilizada por esses pacientes, a maior parte dos cirurgiõesdentistas relata se comunicar principalmente por gestos e leitura labial, visto que não estão aptos para a comunicação em $\operatorname{Libras}^{19,23}$, além de geralmente solicitarem a presença de um intérprete ou familiar ouvinte 21,23. $^{2}$.

Estudos apontam que os acadêmicos de Odontologia e cirurgiões-dentistas consideram importante essa comunicação e possuem interesse em estudar Libras, mas julgam-se despreparados por não terem realizado cursos preparatórios para o atendimento desses pacientes ${ }^{23,24}$. Quando questionados sobre como melhorar esse quadro, acreditam que a oferta de cursos de Libras seja capaz de acrescentar qualidade ao atendimento desse público ${ }^{23-25}$. Essa capacitação inicial pode ser alcançada na formação acadêmica tanto pela oferta 
da disciplina de Libras como componente curricular para todos os cursos de saúde, quanto por meio de projetos de extensão, iniciação científica, entre outros projetos que abordem a temática de acessibilidade no atendimento de surdos. Além disso, essa qualificação pode ser alcançada também após a graduação, por meio de cursos de formação continuada na área ${ }^{19,26}$.

A inclusão da disciplina de Libras nas IES, aliada a projetos extracurriculares, colabora também para a criação e desenvolvimento de novos termos odontológicos específicos em Libras e consequentemente para maior aprimoramento didático-científico dessa área, com a finalidade de potencializar o ensino e a formação de cirurgiõesdentistas. A partir disso, será possível fomentar e ampliar o processo de acessibilidade do atendimento do público surdo ${ }^{26,27}$.

A necessidade da inclusão dessa disciplina nas matrizes curriculares foi discutida também em outras áreas da saúde. Guarinello et $a l^{28}$ verificaram a inclusão da disciplina de Libras em 7 cursos de graduação em Fonoaudiologia, as quais eram ofertadas de forma obrigatória em todas as IES. Essa divergência pode ser explicada a partir do Decreto $\mathrm{n}^{\mathrm{o}} 5.626 / 05^{3}$, o qual determinou a obrigatoriedade de oferta da disciplina, com prazo de até 2015 para sua regularização. A carga horária analisada teve média de $60,8 \mathrm{~h}$ e, embora seja uma média de $11 \mathrm{~h}$ maior que a observada no presente estudo, $158(65,8 \%)$ estudantes a consideraram insuficiente.

Embora os dados sejam expressivos, sugerese que sejam investigados utilizando também formas alternativas de coleta de dados, como a utilização de questionários enviados diretamente aos coordenadores dos cursos de graduação em Odontologia das IES brasileiras, aprimorando a compreensão desse quadro de ensino e verificando outras variáveis, como o período em que é ministrada, conteúdos abordados e a formação do docente responsável. Além disso, é importante destacar como limitação deste estudo, assim como outros de metodologias semelhantes ${ }^{29}$, que em algumas instituições este componente curricular pode ser ministrado em outras disciplinas, como por exemplo, Odontologia para Pacientes com Necessidades Especiais.

\section{CONCLUSÃO}

O presente estudo possibilitou traçar um panorama da inserção da disciplina de Libras nas matrizes curriculares dos cursos de Odontologia da região Sudeste do Brasil, mostrando que sua implementação ainda é discreta nas IES públicas e mais presente nas privadas. Em ambos os casos, a oferta é, majoritariamente, não-obrigatória.

\section{ABSTRACT \\ Brazilian Sign Language teaching in Dentistry undergraduate courses at Southeastern Brazil: a cross-sectional study}

Dental care for deaf patients is considered a challenge. The lack of students' contact with the Brazilian Sign Language (BSL) during graduation can be an aggravating factor of the oral health condition of this population. Thus, the objective of the study was to offer an overview of the inclusion of the BSL subject in the pedagogical projects of undergraduate courses of Dentistry in the southeastern region of Brazil. It consists of an exploratory, quantitative and crosssectional study. The curricular matrices of the courses were analyzed, searching for information regarding the following variables: administrative category of the Higher Education Institution (HEI), offer and nature of the curricular component, and workload. The study population consisted of 176 HEIs, of which 32 did not provide their respective curricular matrices. It was observed that 125 courses $(86.8 \%)$ were from private HEIs and 19 (13.19\%) from public ones, with $83(57.63 \%)$ offering the BSL curriculum component in its matrix, with 71 (91, $02 \%$ ) of the 78 HEIs that provided the information offer the content as optional or elective. The workload varied from 30 to 80 
hours, with an average of 49.7 hours. It is concluded that the implementation of BSL is still discreet in public universities and more concentrated in private HEIs, being, and in its majority, non-mandatory.

Descriptors: Education, Dental. Sign Language. Curriculum.

\section{REFERÊNCIAS}

1. IBGE. Censo Demográfico 2010: características gerais da população, religião e pessoas com deficiência. Rio de Janeiro, 2010.

2. Skliar C. Uma Perspectiva Sócio-Histórica sobre a Psicologia e a Educação dos Surdos. In: Skliar C. Educação e Exclusão. Porto Alegre: Mediação, 1997. p. 105-153

3. Brasil. Decreto $n^{\circ} 5.626$, de 22 de dezembro de 2005. Brasília: DF; 2005 [acesso em 7/07/2019]. Disponível em: http://www. planalto.gov.br/ccivil_03/_Ato2004-2006 /2005/Decreto/D5626.htm.

4. Brasil. Lei no 10.436, de 24 de abril de 2002. Brasília: DF; 2002 [acesso em 28/07/2019]. Disponível em: http://www.planalto.gov.br/ ccivil_03/leis/2002/110436.htm.

5. Brasil, Ministério da Educação. Conselho Nacional de Educação. Câmara de Educação Superior. Diretrizes Curriculares Nacionais do Curso de Graduação em Odontologia. Diário Oficial da União. Brasília: DF; 2018 [acesso em 16/07/2019]. Disponível em: http://portal.mec.gov.br/docman/abril-2019pdf/111231-pces803-18/file.

6. Martins VRO. Análise das vantagens e desvantagens da Libras como disciplina curricular no ensino Superior. Rev Cad CEOM. 2008;21(28):191-206.

7. Al-Qahtani Z, Wyne AH. Caries experience and oral hygiene status of blind, deaf and mentally retarded female children in Riyadh, Saudi Arabia. Odontostomatol Trop. 2004;27(105):37-40.
8. Doichinova L, Peneva M. Prevalence of dental caries in hearing impaired children than 5 to 12 years old in Sofia. IJSC. 2015;4(1):1088-91.

9. Moller CC, Ibaldo LTS, Tovo MF. Avaliação das condições de saúde bucal de escolares deficientes auditivos no município de Porto Alegre, RS, Brasil. Pesqui Bras Odontopediatria Clín Integr. 2010;10(2):195-200.

10. Martin ASS, Chisini LA, Martelli S, Sartori LRM, Ramos EC, Demarco FF. Distribuição dos cursos de Odontologia e de cirurgiõesdentistas no Brasil: uma visão do mercado de trabalho. Rev ABENO. 2018;18(1):63-73.

11. Brasil. Ministério da Educação. Cadastro eMEC de Instituições e Cursos de Educação Superior. Brasília: DF [acesso em 8/07/2019]. Disponível em: http://emec. mec.gov.br/.

12. Brasil. Ministério da Educação. Portaria Normativa $\mathrm{n}^{\circ} 21$, de 21 de dezembro de 2017. Diário Oficial da União. Brasília: DF [acesso em 8/07/2019]. Disponível em: http://www.in.gov.br/materia/-/asset publisher/Kujrw0TZC2Mb/content/id/1284 644/do1-2017-12-22-portaria-n-21-de-21de-dezembro-de-2017-1284640-1284640.

13. IBGE. Diretoria de Pesquisas - DPE Coordenação de População e Indicadores Sociais - COPIS. 2018.

14. Oliveira YCA, Costa GMC, Coura AS, Cartaxo RO, França ISX. A língua brasileira de sinais na formação dos profissionais de enfermagem, fisioterapia e odontologia no estado da Paraíba, Brasil. Interface. 2012;16(43):995-1008.

15. Alsmark SSB, García JN, Martínez MRM, López NEG. How to improve communication with deaf children in the dental clinic. Med Oral Patol Oral y Cir Bucal. 2007;12(8):576-81. 
16. Novaes MSP. Atenção odontológica integral a deficientes auditivos: uma proposta. [Tese]. São Paulo: Faculdade de Odontologia da Universidade de São Paulo; 1997.

17. Chaveiro N, Porto CC, Barbosa MA. Relação do paciente surdo com o médico. Rev Bras Otorrorrorrinolaringol. 2009; 75(1):147-50.

18. Champion J, Holt R. Paediatric dentistry: Dental care for children and young people who have a hearing impairment. Br Dent J. 2000;189(3):155-9.

19. Pereira RM, Monteiro LPA, Monteiro ACC, Costa ICC. Percepção das pessoas surdas sobre a comunicação no atendimento odontológico. Rev Ciênc Plur. 2017;3(2):53-72.

20. Alshehri AA, Togoo RA, Ain TS. Dental treatment experiences of patients with hearing impairment in southern saudi arabia: a questionnaire based study. J Clin Diagn Res. 2018;12(3):ZC37-ZC41.

21. Aragão JS, Magalhães IMO, Coura AS, Silva AFR, Cruz GKP, França ISX. Acesso e comunicação de adultos surdos: uma voz silenciada nos serviços de saúde. Rev Pesqui Cuid Fundam. 2014;6(1):1-7.

22. Patcharaphol S. Dental cares for patients who have a hearing impairment. Int J Clin Prev Dent. 2014;10(4):215-8

23. Silva MC, Rodrigues W. Acessibilidade no tratamento odontológico do paciente surdo. Rev CROMG. 2018;16(1):12-8.
24. Ramos TS, Almeida MAPT. A Importância do ensino de Libras: Relevância para Profissionais de Saúde. Id On Line. 2017;10(33):116-26.

25. Freire DB, Gigante LP, Béria JU, Palazzo LS, Figueiredo ACL, Raymann BCW. Acesso de pessoas deficientes auditivas a serviços de saúde em cidade do sul do Brasil. Cad Saúde Pública. 2009;25(4):889-97.

26. Souza MT, Porrozzi R. Ensino de Libras para os profissionais de saúde: uma necessidade premente. Rev Práxis. 2009;1(2):43-6.

27. Silva LS, Leal JGG, Junior GS, Silva MAD, Pereira AC. Sinais específicos em Libras para o ensino odontológico. Rev ABENO. 2018;18(2):135-43.

28. Guarinello AC, Berberian AP, Eyng DB, Festa PSV, Marques JM, Bortolozzi KB. A disciplina de Libras no contexto de formação acadêmica em fonoaudiologia. Rev CEFAC. 2013;15(2):334-40.

29. Faria LV, Oliveira GA, Grázzia MEP, Medeiros YL, Lopes DF, Leite ICG. O ensino de implantodontia nas graduações brasileiras de Odontologia: um estudo transversal. REAS. 2020; 12(4):e2672.

\section{Correspondência para:}

Yuri de Lima Medeiros

e-mail: yuri11medeiros@gmail.com

Rua Adolpho Kirchmaier, 42 - São Pedro

36036-630 Juiz de Fora/MG 